Received 20.11.2013 Reviewed 09.01.2014 Accepted 16.01.2014

A - study design $\mathbf{B}$ - data collection C - statistical analysis

D - data interpretation

E - manuscript preparation

$\mathbf{F}$ - literature search

\section{Protection of floristic diversity in the Sajna River valley - in view of the planned construction of hydroelectric power station in Sarkajmy}

\author{
Grażyna LASKA ABDEF
}

Białystok Technical University, Department of Environmental Protection and Management, Wiejska 45a, 15-351 Białystok, Poland; e-mail: prorektor.dydaktyka@pb.edu.pl

For citation: Laska G. 2014. Protection of floristic diversity in the Sajna River valley - in view of the planned construction of hydroelectric power station in Sarkajmy. Journal of Water and Land Development. No. 20 p. 45-56.

\begin{abstract}
The study was carried out to determine the floristic diversity in the Sajna River valley, in the region of direct and indirect impact of the hydroelectric power station planned to be built in the village of Sarkajmy. Phytosociological diversity of plant communities and floristic diversity were analysed taking into account the predicted influence of the planned power station on the Sajna River. The study was performed in 2010 using field methods and GIS localisation of analysed sites. 73 phytosociological surveys were made and particular floristic patches were identified. Global Positioning System (GPS) surveys were used to delimit the different plant communities and spot the sites of protected plant species.

Thirteen plant communities were identified in the study area, five of which are of European importance that require protection within Natura 2000 sites. This group of plant communities comprises four forest plant communities (Salicetum albo-fragilis, Ficario-Ulmetum, Tilio-Carpinetum, Acer platanoides-Tilia cordata) and one herbaceous community (Urtico-Calystegietum). One species under strict protection (Daphne mezereum L.) and five species under partial protection (Viburnum opulus L., Ribes nigrum L., Asarum europaeum L., Eurhynchium angustirete (Broth.) T.J.Kop., Eurhynchium striatum (Schreb. ex Hedw.) Schimp.) were noted. The presence of valuable natural habitats and protected species in the Sajna River valley indicates the natural potential of the area and illustrates its biological diversity. The studied area is threatened by negative impact of the hydroelectric power station planned to be built on the Sajna River, since water damming will flood the river terrace that will lead to the formation of a 7.25 ha pond of.
\end{abstract}

Key words: hydroelectric power station, Natura 2000, natural habitats, protected plant species, Sajna River valley

\section{INTRODUCTION}

Hydroelectric energy that use the energy of water flow, waves, ocean currents and tides is an important component of the renewable energy. Its contribution to the world power production reaches $20 \%$ [CIECHANOWICZ 2005]. The contribution of hydroelectric energy to the total power production in Poland is just 1.1\% [GORCZYCA 2011]. According to the Climate and Energy Package, approved in 2008 by the European Parliament, the EU member states should in- crease the increase the energy produced from renewable sources up to $20 \%$ till 2020 [Directive 2009/28/ EC]. For Poland, such an increase means an increase in the number of small hydroelectric power plants to about 130 and an increase of the hydroelectric energy produced from $156.9 \mathrm{GWh}$ to $188.3 \mathrm{GWh}$ [CZEKALSKI 2008].

Alternative energy sources should bring a real chance to the protection of natural resources for future generations and must contribute to environmental protection in general [NOWICKI 2004]. However, the con- 
struction of a hydroelectric power station may considerably threaten local ecosystems, thus the interruption of the river flow and flooding by damming may bring more harm than benefits. The protection of natural resources of the river valleys are of great importance for biological diversity [MIODUSZEWSKI, OKRUSZKO 2012]. Regulation of the river banks and interference in the hydrological regime may lead to irreversible loss of biological richness and its natural variation [KARAVAN et al. 2013; ŁASKA 2009; 2012; MIODUSZEWSKI 2006; 2012; WARDA et al. 2013]. The aim of the study was to evaluate the current status of floristic diversity in the Sajna River valley and to estimate the potential impact of the construction of a small hydroelectric station on plant communities and species.

\section{STUDY AREA AND METHODS}

The area planned for the construction of hydroelectric power station on the Sajna River is situated in warmińsko-mazurskie voivodeship, Korsze commune, in the village Sarkajmy. The area is located north of the country road 592, Kętrzyn-Bartoszyce, at $6.0 \mathrm{~km}$ from the city of Korsze and about $3.0 \mathrm{~km}$ from the southern border of the Special Protection Area (SPAs) for birds, called Warmińska Refuge PLB280015 [Natura 2000 undated]. The Sajna River valley is partly located in the Special Protection Area of the Guber River valley [Rozporządzenie nr 157... 2008].

The methods used in the work included field studies, geographic information system (GIS) data and global positioning system (GPS) data collection. Field surveys in the Sajna River valley were performed in two time periods: in spring (29.04-02.05. 2010) taking into account the appearance of geophytes, and in summer at the peak of the vegetation season (12-14.08.2010). The data were collected from direct and indirect impact area of the planned hydroelectric power station (up to $250 \mathrm{~m}$ ). The field surveys included cartographic work, phytosociological documentation of plant communities and identification of the habitat types characterised by particular plant patches. Seventy three phytosociological surveys were made using the Braun-Blanquet 6-degree coverage-abundance scale on sample plots of $200 \mathrm{~m}^{2}(10 \times$ $20 \mathrm{~m}), 100 \mathrm{~m}^{2}(10 \times 10 \mathrm{~m})$ or $50 \mathrm{~m}^{2}(5 \times 10 \mathrm{~m})$, depending on the size and biological diversity of the studied plant communities. Each sample plot was characterised by its vertical structure and floristic composition. The syntaxonomy of the natural vegetation units was adopted after MATUSZKIEWICZ [2001]. The natural habitats listed in the Annex I of the Habitat Directive were coded according to HERBICH [2004].

GPS was used to locate the study sites, the areas covered by particular plant communities, which were delimited and the sites occupied by protected plant species were spotted. GIS allowed the graphical rep- resentation of the plant communities spatial distribution as a vegetation communities map.

\section{RESULTS AND DISCUSSION}

\subsection{PLANNED CONSTRUCTION}

The construction place of the hydroelectric station is located at $12+330 \mathrm{~km}$ of the Sajna River in the village Sarkajmy, the land plots no. 19-1 from Krzemity Range and no. 103 from Sarkajmy Range. A fragment of $1.25 \%$ of plot no. 19-1 (5.28 ha) will be used for the construction. The stage of fall and a three flanking pier weir $8 \mathrm{~m}$ wide will be built together with the first block of hydroelectric power station of the area of $90 \mathrm{~m}^{2}$ and a $8.6 \mathrm{~m}$ wide $45.0 \mathrm{~m}$ long discharge channel. A fragment of $3.6 \%$ of the total area of 0.25 ha land plot no. 103 i.e. $90 \mathrm{~m}^{2}$ (road) is planned to be taken for the second block of the hydroelectric power station. The hydroelectric power station will have two Kaplan turbines generating power of $0.082 \mathrm{MW}$. The ordinate of dam is $50.5 \mathrm{~m}$ a.s.1., while the height of damming is $4.10 \mathrm{~m}$. If the hydroelectric power station of the above parameters is built, river water will make a water reservoir of the mean depth of $1.20 \mathrm{~m}$, the area of 7.25 ha and total capacity of $87000 \mathrm{~m}^{3}$, flooding the Sajna River valley and plant communities and arable lands near the valley. The land is partly in private hands and partly state owned [KASKA et al. 2010].

The area subject to direct impact of the power station includes a section of the Sajna River bed and its flooding terrace covered with vegetation. The vegetation will be destroyed, trees along the road to the power station and along the discharge channel will have to be cut. River banks and its bottom will have to be strengthened with concrete blocks or gabions along $50 \mathrm{~m}$ below the dam and embankment of the water reservoir.

\section{FLORISTIC DIVERSITY OF PLANT COMMUNITIES}

Thirteen plant communities were identified in the Sajna River valley and in direct and indirect impact areas (Tab. 1) including eight herbaceous communities and five forest communities representing six phytosociological classes. Among them are five plant communities that require protection according to Natura 2000 regulations as specified in Annex I of the Council Directive 92/43/EEG of May 1992 regarding the conservation of the natural habitats and wild fauna and flora. From the above mentioned Natura 2000 protected habitats four are forest communities: riparian willow with Salix fragilis L. representing the community of Salicetum albo-fragilis R.Tx. 1955 (code 91E0-1), ash-elm floodplain forest FicarioUlmetum minoris Knapp 1942 em. J.Mat. 1976 (code 91F0-2), oak-lime-hornbeam forest Tilio cordataeCarpinetum betuli Tracz. 1962 (code 9170-2) and 
Table 1. Floristic diversity of plant communities in the Sajna River valley in 2010 year

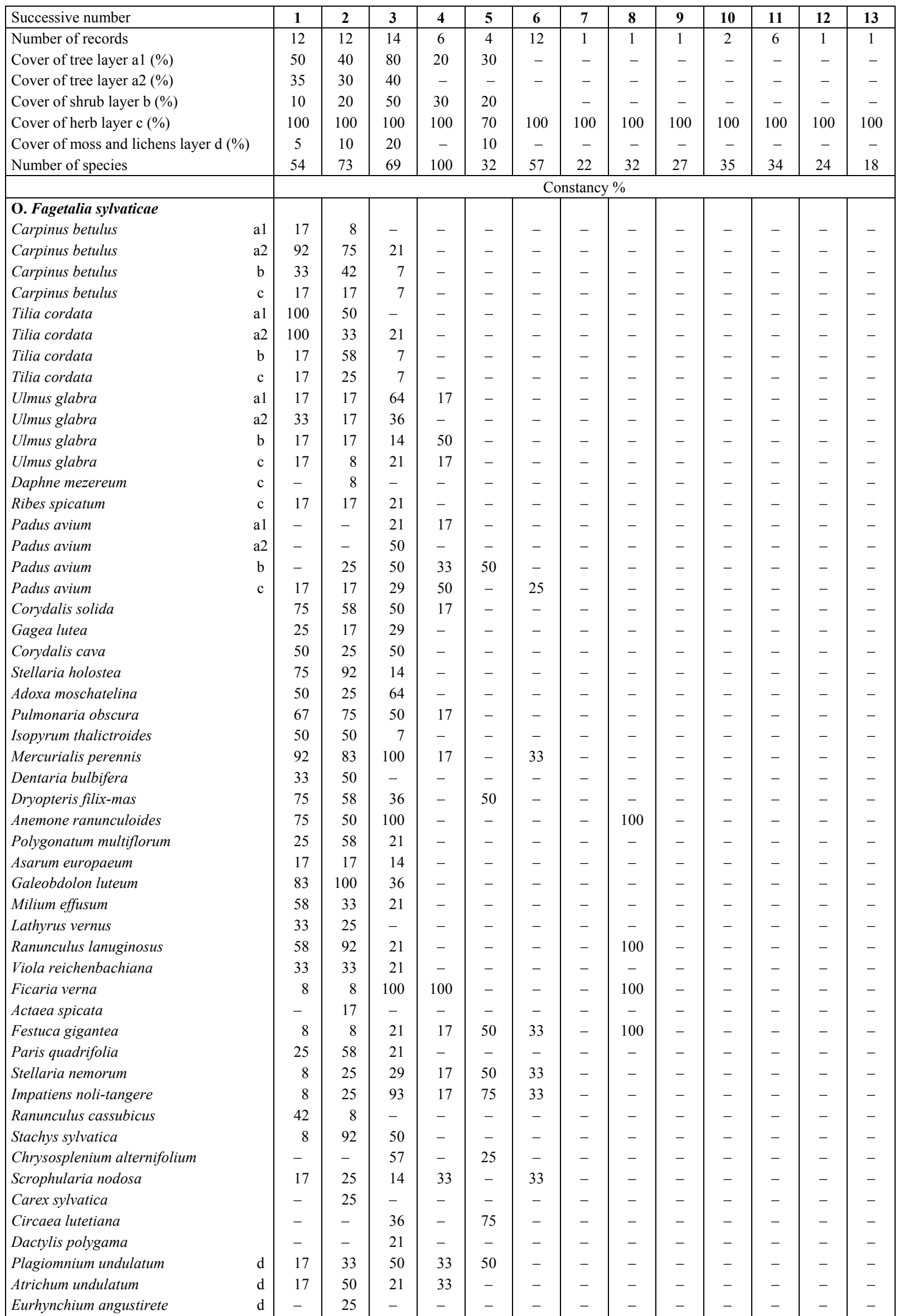


continue Tab. 1

\begin{tabular}{|c|c|c|c|c|c|c|c|c|c|c|c|c|c|c|}
\hline \multicolumn{2}{|l|}{ Successive number } & 1 & 2 & 3 & 4 & 5 & 6 & 7 & 8 & 9 & 10 & 11 & 12 & 13 \\
\hline Eurhynchium striatum & $\mathrm{d}$ & - & 17 & - & - & - & - & - & - & - & - & - & - & - \\
\hline \multicolumn{15}{|l|}{ Cl. Querco-Fagetea } \\
\hline Fraxinus excelsior & a1 & 17 & 83 & 57 & 17 & - & - & - & - & - & - & - & - & - \\
\hline Fraxinus excelsior & a2 & 75 & 17 & 14 & - & - & - & - & - & - & - & - & - & - \\
\hline Fraxinus excelsior & $\mathrm{b}$ & 17 & 17 & 14 & 50 & - & - & - & - & - & - & - & - & - \\
\hline Fraxinus excelsior & $\mathrm{c}$ & 17 & 17 & 29 & 17 & - & - & - & - & - & - & - & - & - \\
\hline Acer platanoides & a1 & 100 & 58 & 29 & 17 & - & - & - & - & - & - & - & - & - \\
\hline Acer platanoides & a2 & 100 & 17 & 7 & - & - & - & - & - & - & - & - & - & - \\
\hline Acer platanoides & $\mathrm{b}$ & 17 & 17 & 7 & - & - & - & - & - & - & - & - & - & - \\
\hline Acer platanoides & $\mathrm{c}$ & 50 & 33 & 14 & 50 & - & - & - & - & - & - & - & - & - \\
\hline Acer campestre & a1 & 33 & 8 & - & - & - & - & - & - & - & - & - & - & - \\
\hline Acer campestre & $\mathrm{a} 2$ & 17 & - & 14 & - & - & - & - & - & - & - & - & - & - \\
\hline Euonymus europaea & $\mathrm{b}$ & 17 & 17 & 7 & - & - & - & - & - & - & - & - & - & - \\
\hline Euonymus europaea & $\mathrm{c}$ & 8 & 8 & 14 & - & - & - & - & - & - & - & - & - & - \\
\hline Euonymus verrucosa & $\mathrm{b}$ & 50 & 25 & 36 & - & - & - & - & - & - & - & - & - & - \\
\hline Euonymus verrucosa & $\mathrm{c}$ & 25 & 8 & 29 & - & - & - & - & - & - & - & - & - & - \\
\hline Corylus avellana & $\mathrm{b}$ & 100 & 83 & 50 & 50 & - & - & - & - & - & - & - & - & - \\
\hline Corylus avellana & $\mathrm{c}$ & 50 & 67 & 43 & - & - & - & - & - & - & - & - & - & - \\
\hline Lonicera xylosteum & $\mathrm{b}$ & 8 & 8 & - & - & - & - & - & - & - & - & - & - & - \\
\hline Lonicera xylosteum & $\mathrm{c}$ & 17 & 8 & - & - & - & - & - & - & - & - & - & - & - \\
\hline Aegopodium podagraria & & 83 & 100 & 57 & 100 & 50 & 100 & - & 100 & - & 50 & - & - & - \\
\hline Anemone nemorosa & & 50 & 75 & 86 & - & - & - & - & - & - & - & - & - & - \\
\hline Melica nutans & & 17 & 17 & 7 & - & - & - & - & - & - & - & - & - & - \\
\hline Poa nemoralis & & 17 & 33 & 21 & - & - & - & - & - & - & - & - & - & - \\
\hline \multicolumn{15}{|l|}{ Cl. Salicetea purpureae } \\
\hline Salix fragilis & a & - & - & - & 83 & - & - & - & - & - & - & - & - & - \\
\hline Salix fragilis & $\mathrm{b}$ & - & - & - & 100 & - & - & - & - & - & - & - & - & - \\
\hline Salix fragilis & $\mathrm{c}$ & - & - & - & 67 & - & 42 & - & - & - & - & - & - & - \\
\hline \multicolumn{15}{|l|}{ DCl. } \\
\hline Stachys palustris & & - & - & - & 67 & - & 25 & - & - & - & - & - & 100 & - \\
\hline \multicolumn{15}{|l|}{ All. Salicion albae } \\
\hline Salix triandra ssp. discolor & $\mathrm{b}$ & - & - & - & 33 & - & - & - & - & - & - & - & - & - \\
\hline Salix viminalis & $\mathrm{b}$ & - & - & - & 33 & - & - & - & - & - & - & - & - & - \\
\hline Salix viminalis & $\mathrm{c}$ & - & - & - & 50 & - & 50 & - & 100 & - & - & - & - & - \\
\hline \multicolumn{15}{|l|}{ DAll. } \\
\hline Calystegia sepium & & - & - & - & 100 & - & 100 & - & - & - & - & - & - & 100 \\
\hline Humulus lupulus & & - & - & - & 83 & - & 100 & - & - & - & - & - & - & 100 \\
\hline Phalaris arundinacea & & - & - & - & 83 & - & 33 & - & - & - & 100 & - & 100 & 100 \\
\hline Rubus caesius & & - & - & - & 100 & - & 75 & - & - & - & - & - & - & - \\
\hline Symphytum officinale & & - & - & - & 50 & - & - & - & - & - & - & - & - & - \\
\hline \multicolumn{15}{|l|}{ Cl. Phragmitetea } \\
\hline Glyceria maxima & & - & - & - & - & - & 17 & - & - & - & - & - & - & 100 \\
\hline Phragmites australis & & - & - & - & 33 & 100 & 25 & - & 100 & - & - & - & 100 & - \\
\hline Carex acutiformis & & - & - & - & 33 & 100 & - & - & - & - & - & - & 100 & - \\
\hline Typha latifolia & & - & - & - & - & - & - & - & - & - & - & - & 100 & 100 \\
\hline Carex vesicaria & & - & - & - & 17 & - & - & - & 100 & - & - & - & - & - \\
\hline Iris pseudoacorus & & - & - & - & 17 & 100 & 17 & - & 100 & - & - & - & 100 & - \\
\hline Poa palustris & & - & - & - & - & - & - & - & - & - & - & - & 100 & 100 \\
\hline Rumex hydrolapathum & & - & - & - & - & - & 25 & - & - & - & - & - & 100 & - \\
\hline Rorippa amphibia & & - & - & - & - & - & - & - & - & - & - & - & 100 & - \\
\hline \multicolumn{15}{|l|}{ Cl. Alnetea glutinosae } \\
\hline Alnus glutinosa & a1 & - & 33 & 21 & 17 & 100 & - & - & - & - & - & - & - & - \\
\hline Alnus glutinosa & $\mathrm{a} 2$ & - & 8 & 21 & - & - & - & - & - & - & - & - & - & - \\
\hline Alnus glutinosa & $\mathrm{b}$ & - & - & - & - & 25 & - & - & - & - & - & - & - & - \\
\hline Alnus glutinosa & $\mathrm{c}$ & - & 8 & 21 & - & 25 & - & - & - & - & - & - & - & - \\
\hline Betula pendula & $\mathrm{b}$ & 8 & - & - & - & 25 & - & - & - & - & - & - & - & - \\
\hline Betula pendula & $\mathrm{c}$ & 8 & - & 7 & - & 25 & - & - & - & - & - & - & - & - \\
\hline Salix cinerea & $\mathrm{b}$ & - & - & - & 33 & 25 & - & - & - & - & - & - & - & - \\
\hline Salix cinerea & $\mathrm{c}$ & - & - & - & 33 & 25 & 25 & - & 100 & - & - & - & - & - \\
\hline Ribes nigrum & $\mathrm{c}$ & - & - & 21 & - & 100 & - & - & - & - & - & - & - & - \\
\hline Lycopus europaeus & & - & - & - & - & 50 & 25 & - & 100 & - & - & - & - & - \\
\hline
\end{tabular}


continue Tab. 1

\begin{tabular}{|c|c|c|c|c|c|c|c|c|c|c|c|c|c|c|}
\hline \multirow{2}{*}{\multicolumn{2}{|c|}{ Successive number }} & 1 & 2 & 3 & 4 & 5 & 6 & 7 & 8 & 9 & 10 & 11 & 12 & 13 \\
\hline & & \multicolumn{13}{|c|}{ Cl. Molinio-Arrhenatheretea } \\
\hline Angelica sylvestris & & 17 & 17 & - & 33 & - & 33 & 100 & - & 100 & - & - & 100 & - \\
\hline \multicolumn{2}{|l|}{ Deschampsia caespitosa } & - & - & 14 & 17 & - & 33 & 100 & - & 100 & 100 & 33 & 100 & - \\
\hline \multicolumn{2}{|l|}{ Lysimachia vulgaris } & - & - & - & 33 & 50 & 25 & - & - & 100 & - & - & 100 & - \\
\hline \multicolumn{2}{|l|}{ Ranunculus repens } & - & - & - & 50 & 50 & 50 & 100 & 100 & 100 & 100 & 100 & - & - \\
\hline \multicolumn{2}{|l|}{ Crepis paludosa } & - & - & - & - & 50 & - & - & - & - & - & - & - & - \\
\hline \multicolumn{2}{|l|}{ Phleum pretense } & - & - & - & - & - & - & 100 & - & - & 100 & 50 & - & - \\
\hline \multicolumn{2}{|l|}{ Lolium perenne } & - & - & - & - & - & - & - & - & - & - & 33 & - & - \\
\hline \multicolumn{2}{|l|}{ Festuca rubra } & - & - & - & - & - & - & - & - & - & - & 33 & - & - \\
\hline \multicolumn{2}{|l|}{ Carex hirta } & - & - & - & 50 & - & 33 & - & - & - & 100 & 67 & - & - \\
\hline \multicolumn{2}{|l|}{ Juncus effusus } & - & - & - & 33 & - & - & 100 & 100 & 100 & - & - & 100 & - \\
\hline Prunella vulgaris & & - & - & - & 17 & - & - & - & - & 100 & 50 & - & - & - \\
\hline Leontodon autumnalis & & - & - & - & - & - & - & - & - & - & 100 & - & - & - \\
\hline Juncus conglomeratus & & - & - & - & - & - & - & 100 & 100 & 100 & & 33 & 100 & - \\
\hline Cirsium oleraceum & & - & - & - & - & 75 & 50 & 100 & 100 & 100 & & 33 & - & - \\
\hline Achillea millefolium & & - & - & - & 17 & - & 42 & 100 & - & 100 & 100 & 100 & - & - \\
\hline Cerastium holosteoides & & - & - & - & - & - & - & 100 & - & 100 & 100 & 67 & - & - \\
\hline Dactylis glomerata & & - & - & - & 83 & - & 33 & - & - & 100 & 50 & 100 & - & 100 \\
\hline Poa trivialis & & - & - & - & 33 & - & - & 100 & - & 100 & 100 & 83 & - & 100 \\
\hline Vicia cracca & & - & - & - & - & - & - & 100 & - & 100 & 100 & 100 & - & - \\
\hline Taraxacum officinale & & - & 25 & - & 83 & - & - & 100 & - & 100 & 100 & 100 & - & - \\
\hline Geum rivale & & - & 33 & 36 & 100 & 75 & 50 & 100 & 100 & 100 & - & - & - & - \\
\hline Plantago lanceolata & & - & - & - & 33 & - & - & 100 & 100 & - & 100 & 50 & - & - \\
\hline Plantago major ssp. major & & - & - & - & 17 & - & - & 100 & 100 & - & - & 33 & - & - \\
\hline Poa pratensis & & - & - & - & 17 & - & - & - & - & 100 & 100 & 33 & - & - \\
\hline Ranunculus acris & & - & - & - & 33 & - & - & 100 & - & 100 & 100 & 100 & - & - \\
\hline Rumex acetosa & & - & - & - & 33 & - & - & 100 & - & 100 & 100 & 50 & - & - \\
\hline Роа аппиа & & - & - & - & 17 & - & - & - & - & - & 50 & 33 & - & - \\
\hline Alchemilla pastoralis & & - & - & - & 17 & - & - & - & - & - & - & - & - & - \\
\hline Agrostis stolonifera & & - & - & - & 33 & - & - & - & - & - & 100 & 67 & - & - \\
\hline Lysimachia nummularia & & - & - & - & 17 & - & 33 & - & - & - & 50 & & - & - \\
\hline Potentilla anserina & & - & - & - & 67 & 25 & 25 & 100 & 100 & 100 & 100 & 100 & - & - \\
\hline Rumex crispus & & - & - & - & 17 & - & 42 & - & 100 & 100 & & 33 & - & - \\
\hline Scirpus sylvaticus & & - & - & - & 33 & - & 25 & - & 100 & - & - & - & 100 & - \\
\hline Valeriana officinalis & & - & - & - & 17 & - & - & - & - & - & - & - & & - \\
\hline Alopecurus pratensis & & - & - & - & 33 & - & - & 100 & - & 100 & 100 & - & 100 & - \\
\hline Trifolium repens & & - & - & - & 67 & - & - & 100 & 100 & 100 & 100 & 100 & - & - \\
\hline Cardamine pratensis & & - & - & - & 33 & - & 25 & - & - & 100 & - & - & - & - \\
\hline Geranium pratense & & - & - & - & 17 & - & - & - & - & - & - & - & - & - \\
\hline Bromus hordeaceus & & - & - & - & 17 & - & - & - & - & - & - & - & - & - \\
\hline Caltha palustris & & - & - & - & 50 & 100 & - & - & 100 & - & - & - & 100 & - \\
\hline Myosotis palustris & & - & - & - & - & 25 & - & - & - & 100 & - & - & 100 & - \\
\hline Cardamine amara & & - & - & - & - & 50 & - & - & - & & - & - & - & - \\
\hline Holcus lanatus & & - & - & - & - & & - & 100 & - & 100 & 50 & 33 & - & - \\
\hline Stellaria graminea & & - & - & - & - & - & - & - & - & - & 100 & 67 & - & - \\
\hline Cl. Trifolio-Geranietea sangu & & & & & & & & & & & & & & \\
\hline Polygonatum odoratum & & - & 17 & - & - & - & - & - & - & - & - & - & - & - \\
\hline Galium mollugo & & - & - & - & - & - & - & - & - & 100 & - & 100 & - & 100 \\
\hline Trifolium medium & & - & - & - & - & - & - & - & - & - & - & 83 & - & - \\
\hline Cl. Epilobietea angustifolii & & & & & & & & & & & & & & \\
\hline Salix caprea & $\mathrm{b}$ & - & - & - & 17 & - & - & - & - & - & - & - & - & - \\
\hline Salix caprea & $\mathrm{c}$ & - & - & - & 33 & - & - & - & - & - & - & - & - & - \\
\hline Sambucus nigra & $\mathrm{b}$ & 17 & 50 & 50 & 33 & 50 & - & - & - & - & - & - & - & - \\
\hline Sambucus nigra & $\mathrm{c}$ & - & 25 & 14 & 17 & - & 17 & - & - & - & - & - & - & - \\
\hline Rubus idaeus & $\mathrm{c}$ & - & 25 & 36 & 33 & - & - & - & - & - & - & - & - & - \\
\hline Chamaenerion angustifolium & & - & - & - & - & - & 33 & - & - & - & - & - & - & - \\
\hline Cl. Artemisietea vulgaris & & & & & & & & & & & & & & \\
\hline Urtica dioica & & 25 & 75 & 86 & 100 & 75 & 100 & - & - & - & 100 & 33 & 100 & 100 \\
\hline Geum urbanum & & - & 33 & 36 & 67 & - & 33 & - & 100 & - & 50 & 17 & 100 & - \\
\hline Geranium robertianum & & - & 33 & 21 & - & 50 & - & - & - & - & - & - & - & - \\
\hline Epilobium hirsutum & & - & - & - & - & - & - & - & 100 & - & - & - & - & 100 \\
\hline
\end{tabular}


continue Tab. 1

\begin{tabular}{|c|c|c|c|c|c|c|c|c|c|c|c|c|c|c|}
\hline Successive number & & 1 & 2 & 3 & 4 & 5 & 6 & 7 & 8 & 9 & 10 & 11 & 12 & 13 \\
\hline Chelidonium majus & & - & 17 & - & 67 & - & 33 & - & - & - & - & - & - & - \\
\hline Glechoma hederacea & & - & 58 & 36 & 67 & - & 42 & - & 100 & - & 100 & - & - & - \\
\hline Lamium maculatum & & - & - & 21 & - & - & 33 & - & - & - & - & - & - & - \\
\hline Cirsium arvense & & - & - & - & 83 & - & 33 & - & 100 & - & 100 & 50 & 100 & - \\
\hline Cirsium vulgare & & - & - & - & - & - & - & - & - & - & - & 33 & - & - \\
\hline Arctium tomentosum & & - & - & - & 50 & - & 42 & - & 100 & - & - & - & - & - \\
\hline Artemisia vulgaris & & - & - & - & 33 & - & - & - & - & - & - & 33 & - & - \\
\hline Tanacetum vulgare & & - & - & - & 17 & - & - & - & - & - & - & - & - & - \\
\hline Euphorbia cyparissias & & - & - & - & 17 & - & - & - & - & - & - & - & - & - \\
\hline Poa angustifolia & & - & - & - & 33 & - & - & - & - & - & - & - & - & - \\
\hline Hypericum perforatum & & - & - & - & 33 & - & 33 & - & - & - & - & - & - & - \\
\hline Arctium lappa & & - & - & - & 50 & - & 17 & - & - & - & - & - & - & - \\
\hline Leonurus cardiaca & & - & - & - & 17 & - & - & - & - & - & - & - & - & - \\
\hline Rumex obtusifolius & & - & - & - & 50 & - & 50 & - & - & - & 100 & - & 100 & - \\
\hline Ballota nigra & & - & - & - & 17 & - & - & - & - & - & - & - & - & - \\
\hline Galium aparine & & - & - & - & 33 & - & - & - & - & - & - & - & - & - \\
\hline Veronica chamaedrys & & 8 & 17 & 21 & 67 & - & 33 & 100 & - & - & 100 & - & 100 & - \\
\hline Lamium album & & - & - & - & 67 & - & - & - & - & - & - & - & - & - \\
\hline Anthriscus sylvestris & & - & - & - & 33 & - & 33 & - & - & - & - & - & - & - \\
\hline Solidago canadensis & & - & - & - & 33 & - & 33 & - & - & - & - & - & - & - \\
\hline Lythrum salicaria & & - & - & - & 33 & - & 50 & - & 100 & 100 & - & - & 100 & 100 \\
\hline Cl. Stellarietea mediae & & & & & & & & & & & & & & \\
\hline Lapsana communis & & - & - & - & 17 & - & 17 & - & - & - & - & - & - & - \\
\hline Sonchus arvensis & & - & - & - & 17 & - & & - & - & - & - & - & - & - \\
\hline Cl. Rhamno-Prunetea & & & & & & & & & & & & & & \\
\hline Viburnum opulus & $\mathrm{b}$ & - & 8 & - & - & - & - & - & - & - & - & - & - & - \\
\hline Crataegus monogyna & $\mathrm{b}$ & - & - & 29 & 33 & - & - & - & - & - & - & - & - & - \\
\hline Crataegus monogyna & $\mathrm{c}$ & - & - & - & 33 & - & - & - & - & - & - & - & - & - \\
\hline Rosa canina & $\mathrm{c}$ & - & - & - & 17 & - & - & - & - & - & - & - & - & - \\
\hline Rubus caesius & $\mathrm{c}$ & - & - & 36 & - & - & - & - & - & - & - & - & - & - \\
\hline Cornus sanguinea & $\mathrm{b}$ & - & 8 & 29 & - & - & - & - & - & - & - & - & - & - \\
\hline Cornus sanguinea & $\mathrm{c}$ & - & - & 14 & - & - & - & - & - & - & - & - & - & - \\
\hline Cl. Agropyretea intermed & & & & & & & & & & & & & & \\
\hline Agropyron repens & & - & - & - & 17 & - & 33 & - & 100 & - & 100 & - & - & - \\
\hline Bromus inermis & & - & - & - & 17 & - & - & - & - & - & - & - & - & 100 \\
\hline Accompanying species & & & & & & & & & & & & & & \\
\hline Quercus robur & a1 & 42 & 33 & 21 & 17 & - & - & - & - & - & - & - & - & - \\
\hline Quercus robur & $\mathrm{a} 2$ & 17 & - & 14 & - & - & - & - & - & - & - & - & - & - \\
\hline Quercus robur & $\mathrm{c}$ & 17 & 8 & 14 & - & - & - & - & - & - & - & - & - & - \\
\hline Populus tremula & $\mathrm{c}$ & 8 & 17 & 7 & - & - & - & - & - & - & - & - & - & - \\
\hline Malus sylvestris & $\mathrm{b}$ & - & - & - & 17 & - & - & - & - & - & - & - & - & - \\
\hline Malus sylvestris & $\mathrm{c}$ & - & - & - & 17 & - & - & - & - & - & - & - & - & - \\
\hline Acer negundo & a & - & - & - & 33 & - & - & - & - & - & - & - & - & - \\
\hline Acer negundo & $\mathrm{b}$ & - & - & - & 17 & - & - & - & - & - & - & - & - & - \\
\hline Acer negundo & $\mathrm{c}$ & - & - & - & 17 & - & - & - & - & - & - & - & - & - \\
\hline Prunus domestica & $\mathrm{b}$ & - & - & - & 17 & - & - & - & - & - & - & - & - & - \\
\hline Prunus domestica & $\mathrm{c}$ & - & - & - & 33 & - & - & - & - & - & - & - & - & - \\
\hline Aesculus hippocastanum & $\mathrm{a}$ & - & - & - & 17 & - & - & - & - & - & - & - & - & - \\
\hline Aesculus hippocastanum & $\mathrm{c}$ & - & - & - & 17 & - & - & - & - & - & - & - & - & - \\
\hline Oxalis acetosella & & - & - & 21 & - & - & - & - & - & - & - & - & - & - \\
\hline Viola mirabilis & & 75 & 58 & - & - & - & - & - & - & - & - & - & - & - \\
\hline Maianthemum bifolium & & - & 25 & - & - & - & - & - & - & - & - & - & - & - \\
\hline Moehringia trinervia & & - & 25 & - & - & - & - & - & - & - & - & - & - & - \\
\hline Athyrium filix-femina & & - & 25 & 29 & - & 25 & - & - & - & - & - & - & - & - \\
\hline Ajuga reptans & & 8 & 25 & 21 & - & - & - & - & - & - & - & - & - & - \\
\hline Bidens tripartita & & - & - & - & - & - & 33 & - & 100 & - & - & - & - & 100 \\
\hline Polygonum lapathifolium & & - & - & - & - & - & 42 & - & - & - & - & - & - & 100 \\
\hline Rorippa palustris & & - & - & - & - & - & 33 & - & - & - & - & - & - & 100 \\
\hline Capsella bursa-pastoris & & - & - & - & - & - & - & - & - & - & 100 & - & - & - \\
\hline Equisetum sylvaticum & & - & 17 & - & - & - & - & - & - & - & - & - & - & - \\
\hline Mentha arvensis & & - & - & - & 17 & - & 42 & - & 100 & - & 100 & - & - & 100 \\
\hline
\end{tabular}


continue Tab. 1

\begin{tabular}{|c|c|c|c|c|c|c|c|c|c|c|c|c|c|c|}
\hline Successive number & & 1 & 2 & 3 & 4 & 5 & 6 & 7 & 8 & 9 & 10 & 11 & 12 & 13 \\
\hline Agrostis capillaris & & - & - & - & - & - & - & - & - & - & - & 50 & - & - \\
\hline Mentha palustris & & - & - & - & - & - & 33 & - & - & - & - & - & - & 100 \\
\hline Polypodium vulgare & & 17 & - & - & - & - & - & - & - & - & - & - & - & - \\
\hline Cystopteris fragilis & & 17 & - & - & - & - & - & - & - & - & - & - & - & - \\
\hline Comarum palustre & & - & - & - & - & 75 & - & - & - & - & - & - & - & - \\
\hline Plagiomnium affine & d & - & - & 21 & - & 50 & - & - & - & - & - & - & - & - \\
\hline Fissidens taxifolius & d & - & - & 14 & - & - & - & - & - & & - & - & - & - \\
\hline Brachythecium rutabulum & $\mathrm{d}$ & - & - & 21 & 50 & - & - & - & - & - & - & - & - & - \\
\hline
\end{tabular}

Explanations:

1 - Ass. Acer platanoides-Tilia cordata Jutrz.-Trzeb. 1993

2 - Ass. Tilio-Carpinetum Tracz. 1962

3 - Ass. Ficario-Ulmetum minoris Knapp 1942 em. J. Mat. 1976

4 - Ass. Salicetum albo-fragilis R.Tx. 1955

5 - Ass. Ribeso nigri-Alnetum Sol.-Górn. (1975) 1987

6-Ass. Urtico-Calystegietum sepium Görs et Th. Müll. 1969

7 - Community with Deschampsia caespitosa

Source: own study.

slope maple-lime lowland forest Acer platanoides-Tilia cordata Jutrz.-Trzeb. 1993 (code 9170-3), and one nitrophilic community of herbaceous species and bindweeds on the banks of the watercourse Urtico-Calystegietum sepium Görs et Th. Müller 1969 (code 6430-3) (Fig. 1). Syntaxonomy of particular plant taxons used after MATUSZKIEWICZ [2001] is as follows:

Class (Cl.) Salicetea purpureae Moor 1958

Order (O.) Salicetalia purpureae Moor 1958

Alliance (All.) Salicion albae R.Tx. 1955

Association (Ass.) Salicetum albo-fragilis

R.Tx. 1955

Cl. Querco-Fagetea Br.-Bl. et Vlieg. 1937

O. Fagetalia sylvaticae Pawł. in Pawł., Sokoł. et Wall 1928

All. Alno-Ulmion Br.-Bl. et R.Tx. 1943

Ass. Ficario-Ulmetum minoris Knapp 1942 em. J. Mat. 1976

All. Carpinion betuli Issl. 1931 em. Oberd. 1953

Ass. Tilio-Carpinetum Tracz. 1962

Ass. Acer platanoides-Tilia cordata Jutrz.-

-Trzeb. 1993

Cl. Alnetea glutinosae Br.-B1. et R.Tx.1943

O. Alnetalia glutinosae R. Tx.1937

All. Alnion glutinosae (Malc. 1929) Meijer Drees 1936

Ass. Ribeso nigri-Alnetum Sol.-Górn. (1975) 1987

Cl. Artemisietea vulgaris Lohm., Prsg et R.Tx. in R.Tx. 1950

O. Convolvuletalia sepium R.Tx. 1950

All. Convolvulion sepium R.Tx 1947 em Müll. 1981

Ass. Urtico-Calystegietum sepium Görs et Th. Müll. 1969

Cl. Molinio-Arrhenatheretea R. Tx. 1937

O. Molinietalia caeruleae W. Koch 1926

All. Calthion palustris R. Tx. 1936 em. Oberd. 1957

Community with Deschampsia caespitosa Ass. Scirpetum silvatici Ralski 1931
8 - Ass. Scirpetum silvatici Ralski 1931

9 - Ass. Angelico-Cirsietum oleracei R. Tx. 1937 em, Oberd. 1967

10 - Ass. Alopecuretum pratensis (Regel 1925) Steffen 1931

11 - Community with Dactylis glomerata

12 - Ass. Phragmitetum australis (Gams 1927) Schmale 1939

13 - Ass. Glycerietum maximae Hueck 1937

Ass. Angelico-Cirsietum oleracei R. Tx. 1937 em, Oberd. 1967

All. Alopecurion pratensis Pass. 1964

Ass. Alopecuretum pratensis (Regel 1925) Steffen 1931

O. Arrhenatheretalia elatioris Pawł. 1928

Community with Dactylis glomerata

Cl. Phragmitetea R.Tx. et Prsg 1942

O. Phragmitetalia W. Koch 1926

All. Phragmition W. Koch 1926

Ass. Phragmitetum australis (Gams 1927) Schmale 1939

Ass. Glycerietum maximae Hueck 1937

Although the catchment area of the Sajna River is typically agricultural and dominated by arable land, meadows and pastures, the area of direct impact of the planned hydroelectric power station is $90 \%$ forested and has eutrophic and valuable habitats protected within the Natura 2000 programme, specified in Annex I of the Council Directive 92/43/EEG. The greatest part of the area under the direct impact of the station $(40 \%)$ is covered by Ficario-Ulmetum minoris (code 91F0-2) growing on the flooding terrace of the Sajna River, along its eastern and western banks (Fig. 1). These are wet forests growing on highly fertile soils, developed under the influence of flowing water without stagnation tendency. The soil consists of clays, loams or clayey sands. The soil is of black chernozem type or represents certain subtypes of surface-water-gley soil and ground-water-gley soil or brown alluvial soil with deep humus horizon and deep clay horizon. The community is built by multilayer tree stand with a mean degree coverage of $80 \%$, dominated by Fraxinus excelsior L., Ulmus gabra Huds. and Padus avium Mill. (Tab. 1). Rich herbaceous layer has $100 \%$ coverage, includes about 69 plants species dominated by geophytes in spring (Anemone ranunculoides L., Ficaria verna Huds., Corydalis cava Schweigg. \& Körte, C. solida (L.) Clairv.) and hemicryptophytes (Mercurialis perennis L., Asarum europaeum L., Impatiens noli-tangere L.) in summer. 


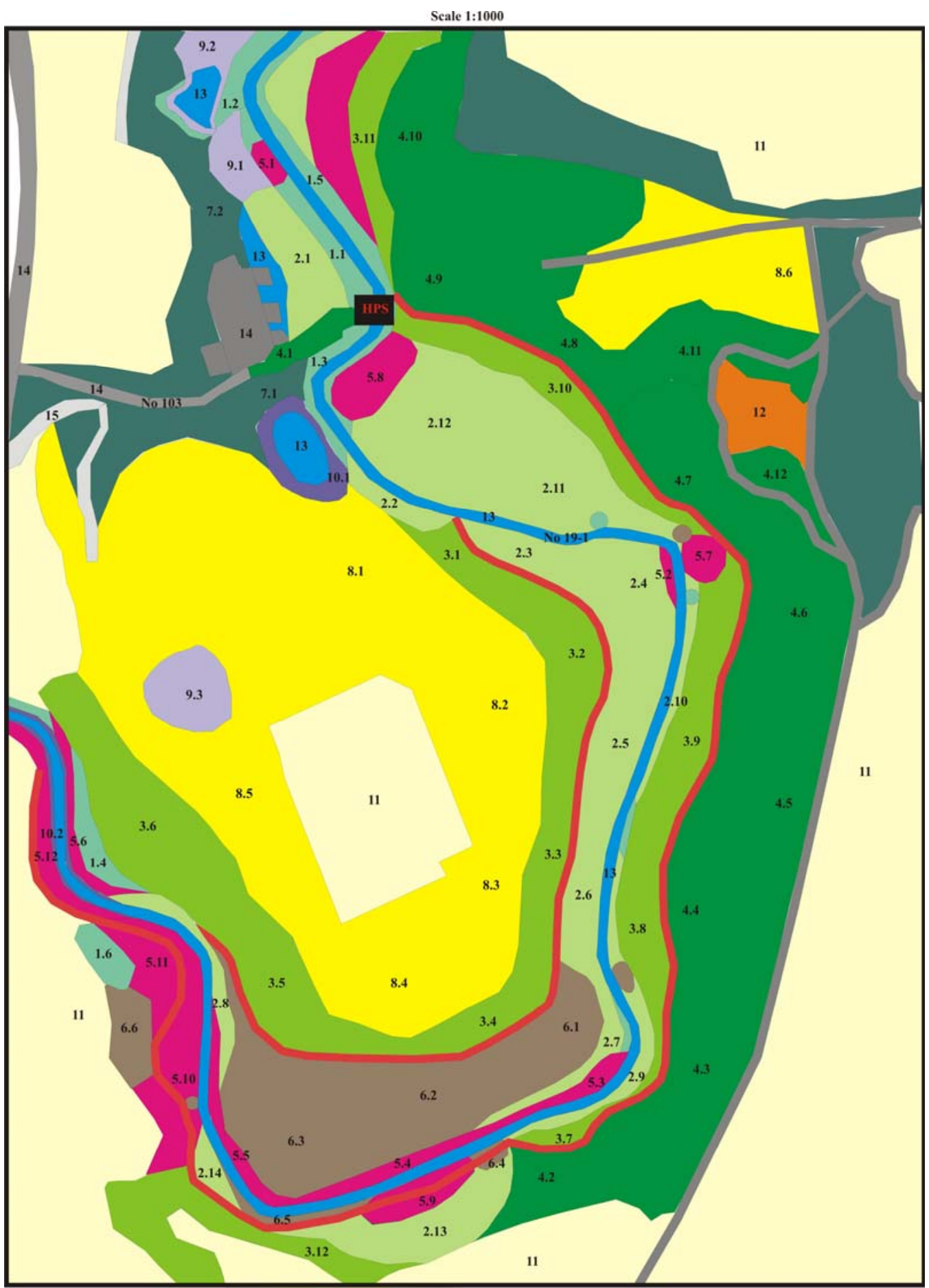

Fig. 1. Distribution of plant communities in the area planned the construction of the hydroelectric power station (HPS) on the Sajna River in the Sarkajmy; source: own elaboration

Ficario-Ulmetum minoris covers the lowest areas on the flood terrace, near the river bed. Above them the river banks are covered with fragments of riparian willow (5\%) with Salix fragilis L. (code 91E0-1). The habitats of riparian willow strech along the river bed, on partially flooded or silt-covered ground. These forests are found in floodable area, mainly at the northern side and mid-western part of the study area, but isolated trees growing along the Sajna River can be noticed there too (Fig. 1). The patches of riparian willow community are dominated by willow trees, mainly Salix fragilis, often represented only by dead fallen logs of old trees. Floristic composition of the herbaceous layer is characterised by high coverage $(80-100 \%)$ and is dominated in general by species belonging to the classes Salicetea purpureae, Phragmitetea, Molinio-Arrhenatheretea and Artemisietea (Tab. 1). Peat bogs without water outflow and habitats of Ribeso nigri-Alnetum occupy the depressions of the Sajna River valley (Fig. 1). 


\section{Legend}

Forest and non-forest plant communities listed in Annex I to the Council Directive 92/43/EEC

3

3. Code 9170-2 - Ass. Tilio cordatae-Carpinetum betuli Tracz. 1962 of the class Querco-Fagetea Br.-Bl. Et Vlieg. 1937 - phytosociological relevés - 3.1-3.12.

4. Code 9170-3 - Ass. Acer platanoides-Tilia cordata Jutrz.-Trzeb. 1993 of the class Querco-Fagetea Br.-BI. et Vlieg. 1937 - phytosociological relevés - 4.1-4.12

5. Code 6430-3 - Ass. Urtico-Calystegietum sepium Görs et Th. Müller 1969 of the subclass Galio-Urticenea Pass. 1967 - phytosociological relevés - 5.1-5.12

Forest and non-forest communities not protected by the Natura 2000

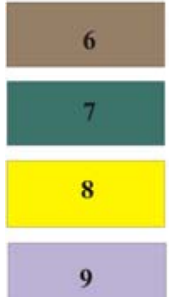

6. Ribeso nigri-Alnetum Sol.-Górn. (1957) 1987 of the class Alnetea glutinosae Br.-Bl. et R.Tx. 1943 - phytosociological relevés - 6.1-6.4

7. Deciduous woodlands and bushes in agricultural fields, strip plantings on roadsides - phytosociological relevés - 7.1-7.2

8. Community with Dactylis glomerata of the class Molinio-Arrhenatheretea R.Tx. 1937 - phytosociological relevés - 8.1-8.6

9. Communities of the All. Calthion palustris R.Tx. $1936 \mathrm{em}$. Oberd. 1957 of the class Molinio-Arrhenatheretea R.Tx. 1937 - phytosociological relevés - 9.1-9.3

10

10. Communities of the class Phragmitetea R.Tx. et Prsg 1942 - phytosociological relevés - 10.1-10.2

11. Agrocenoses - segetal communities of the class Stellarietea mediae R.Tx., Lohm. et Prsg. 1950

12

12. Agrocenoses - fallow, weed communities associated with segetal vegetations of the class Stellarietea mediae R.Tx., Lohm. Et Prsg. 1950

13

13. Water plants communities on the river Sajna, ponds

14

14. Roads, buildings and ruderal communities of the class Artemisietea vulgaris Lohm., Prsg et R.Tx. In R.Tx 1950

15

15. Slopes sand and gravel

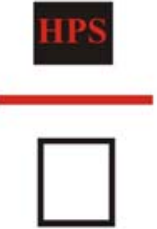

The planned construction of the hydroelectric power station on the river Sajna

1.1

The number of the 73 phytosociological relevés on 73 sample plots

No 19-1

No 103

The numbers of the parcels of the planned construction of the hydroelectric power station on the river Sajna

Ficario-Ulmetum minoris habitat changes into the subcontinental Tilio cordatae-Carpinetum betuli (code 9170-2) (Tab. 1). Tilio-Carpinetum grows at the foot of the upland basal moraine with glacial till, at the eastern and western side of the planned hydroelectric power station $(30 \%)$, in potentially flooded area to be established after the station is built (Fig. 1). Depending on soil moisture, the Tilio-Carpinetum community develops as different subassociations of humid oak-hornbeam forests, ecologically differentiated in
Tilio-Carpinetum corydaletosum, Tilio-Carpinetum mercurialetosum and Tilio-Carpinetum stachyetosum.

The steep or very steep walls of the upland moraine from the indirect impact area of the planned hydroelectric power station, on the eastern and southwestern sides of the river bed (Fig. 1), are dominated by calcareous slope maple forests Acer platanoides-Tilia cordata (code 9170-3) (30\%). These forests grow in clayey sands rich in calcium carbonate or clay periglacial brown soil. The community is character- 
ised by a multispecies tree stand and the tallest layer of trees of $50 \%$ cover is dominated mainly by Acer platanoides L., Quercus robur L. and Tilia cordata Mill. Rich herbaceous layer is characterised by calcareous eutrophic oak-hornbeam wood species from the Fagetalia order and Querco-Fagetea class (Tab. 1).

The herbaceous area in the direct neighbourhood of the planned hydroelectric power station is located mostly on the southern and northern side of the river, very near to the riverbed (Fig. 1). It comprises habitats of nitrophilic herbaceous communities and bindweeds Urtico-Calystegietum sepium (code 6430-3) $(10 \%)$ on the river banks forming the ecotone between the river and forest communities (Tab. 1).

The area of indirect impact of the station, outside the area planned to be flooded in the futures, is mainly covered by arable lands, meadows and pastures (Fig. $1)$. In the norther part of the studied area, there are wet meadows of Calthion palustris association (2\%) and intensively cultivated grasslands representing the class Molinio-Arrhenatheretea (30\%) mostly in the central part of the indirect impact area. There are also segetal communities representing the class Stellarietea mediae $(9 \%)$. They are located in the central part and on western side of the indirect impact area. Small patches are covered by rush $(1 \%)$ and ruderal $(1 \%)$ communities accompanying human dwellings (Fig. 1, Tab. 1).

According to the Directive of the Ministry of Natural Environment of 9 July 2004 [Rozporządzenie MŚ 2004] one species under strict protection and five species under partial protection (including two moss species) were found in the study area (Tab. 2). The planned power station and the wa-ter reservoir will destroy almost all the habitats of the valuable protected species (Fig. 1).

Table 2. Strictly* and partially protected plant species in the study area

\begin{tabular}{|c|l|l|}
\hline No & \multicolumn{1}{|c|}{ Plant species } & \multicolumn{1}{|c|}{\begin{tabular}{c}
\multicolumn{1}{c|}{ Community/ } \\
Phytosociological relevés
\end{tabular}} \\
\hline 1 & Daphne mezereum L. $*$ & $\begin{array}{l}\text { Tilio-Carpinetum corydaletosum/ } \\
\text { relevés 3.9 }\end{array}$ \\
\hline 2 & Ribes nigrum L. & $\begin{array}{l}\text { Ribeso nigri-Alnetum/ relevés 6.1- } \\
6.4 ; \\
\text { Ficario-Ulmetum/ relevés 2.3, 2.4, } \\
2.10\end{array}$ \\
\hline 3 & Asarum europaeum L. & $\begin{array}{l}\text { Ficario-Ulmetum/ relevés 2.3, 2.4; } \\
\text { Tilio-Carpinetum corydaletosum/ } \\
\text { relevés 3.8-3.9 }\end{array}$ \\
\hline 4 & Viburnum opulus L. & $\begin{array}{l}\text { Tilio-Carpinetum corydaletosum/ } \\
\text { relevés 3.9 }\end{array}$ \\
\hline 5 & $\begin{array}{l}\text { Eurhynchium angustirete } \\
\text { (Broth.) T.J.Kop. }\end{array}$ & $\begin{array}{l}\text { Tilio-Carpinetum stachyetosum/ } \\
\text { relevés 3.2 } \\
\text { Tilio-Carpinetum corydaletosum/ } \\
\text { relevés 3.8-3.9 }\end{array}$ \\
\hline 6 & $\begin{array}{l}\text { Eurhynchium striatum } \\
\text { (Schreb. ex Hedw.) } \\
\text { Schimp.) }\end{array}$ & $\begin{array}{l}\text { Tilio-Carpinetum corydaletosum/ } \\
\text { relevés 3.8-3.9 }\end{array}$ \\
\hline
\end{tabular}

Explanation: * - strictly protected plant species.

Source: own study.

\subsection{EVALUATION OF DIRECT AND INDIRECT IMPACT OF PLANNED HYDROELECTRIC POWER STATION}

The planned construction of the hydroelectric power station on the river Sajna implies future formation of a water reservoir occupying an area of about 7.25 ha, which will destroy vegetation of this area. The Sajna River valley belongs to the Protected Area of the River Guber valley according to the Order no. 157 of the Warmian-Masurian Voivode of 19 Dec. 2008 and hence is under active protection of forest, grassland and river ecosystems in the area. Therefore, any investments that could have significant impact on the natural environment are not allowed there. The active protection measures include the setting of compact forest complexes and ecological corridors, conservation and maintenance of watercourses, marshes and wetland areas in a state similar to their natural one in order to increase their biological diversity. Constructions on upland edges and river terraces and any activities that might change the water regime are fully banned [BERNINGER et al. 2012; BUKOWSKI 2013; ILNICKI et al. 2010a, b]. The impact of planned hydroelectric power station and the water reservoir construction will bring the irreversible destruction of the valuable Natura 2000 habitats and sites of protected plant species. Plant communities in the Sajna valley act as an important ecological corridor providing the free flow of genetic resources of the flora and fauna [JANKOWSKA-HUFLEJT et al. 2011].

\section{CONCLUSIONS}

The anthropogenic influence on Sajna River valley is low being reflected in the high variability of plant communities and high floristic diversity. Their existence is critically dependent on the protection of habitat conditions. Preservation of the habitats requires keeping the hydrogeological regime unchanged over the whole catchment area of the Sajna River and its whole hydrological system. The recommended activity should aim at protecting the network of migration corridors for the provision of contacts between plant and animal populations. The corridors link the Protected Area of the Guber River valley and the Sajna River with the Warminska Refuge area protected within the Natura 2000 in one ecological region.

\section{REFERENCES}

Berninger K., Koskiaho J., TAtTari S. 2012. Constructed wetlands in Finnish agricultural environments: balancing between effective water protection, multi-functionality and socio-economy. Journal of Water and Land Development. No 17 p. 19-29.

BUKOWSKI M. 2013. The influence of hydrotechnical conditions on energy production in small-scale hydropower plants. Journal of Water and Land Development. No $18 \mathrm{p}$. 29-35. 
Ciechanowicz W. 2005. Odnawialne źródła energii [Renewable energy sources]. Aura. Nr 8 p. 5-7.

CZEKALSKI A. 2008. Mała energetyka wodna. W: Zarządzanie $\mathrm{w}$ energetyce: koncepcje, zasoby, strategie, struktury, procesy $i$ technologie energetyki odnawialnej [Small water power. In: Energy management: concepts, resources, strategies, structures, processes and renewable energy technologies]. Eds. A. Chochowski, F. Krawiec. Warszawa. Difin p. 238-254.

Directive 2009/28/EC of the European Parliament and of the Council of 23 April 2009 on the promotion of the use of energy from renewable sources and amending and subsequently repealing Directives 2001/77/EC and 2003/30/ EC. OJ L 140/16.

Council Directive 92/43/EEC of 21 May 1992 on the conservation of natural habitats and wild fauna and flora. OJ L 206.

GORCZYCA M. 2011. Energia ze źródeł odnawialnych w Polsce na tle innych krajów Unii Europejskiej [Energy from renewable sources in Poland as compared to other EU countries] [online]. Energetyka i Ekologia p. 515518. [Access 20.11.2013]. Available at: http://energetyka. eu/upload/file/2011/8/Gorczyca.pdf

HerBiCH J. 2004. Poradnik ochrony siedlisk i gatunków Natura 2000 - podręczniki metodyczne [Guide for the protecttion of habitats and species Natura 2000 - Methodology books]. T. 5. Warszawa. MŚ. ISBN 83-86564-43-1. pp. 344.

IlNiCKi P., Górecki K., Grzybowski M., KrZemińska A., LeWANDOWski P., SoJKa M. 2010a. Principles of hydromorphological surveys of Polish rivers. Journal of Water and Land Development. No 14 p. 3-13.

ILNICKI P., GóRECKI K., GRZYBOWSKi M., KRZEMIŃSKA A., LEWANDOWSKI P., SOJKA M. 2010b. Ecological quality classes of river hydromorphology in Poland. Journal of Water and Land Development. No 14 p. 15-27.

JANKOWSKA-Huflejt H., WRÓBEL B., TWARDY S. 2011. Current role of grasslands in development of agriculture and rural areas in Poland - an example of mountain voivodships małopolskie and podkarpackie. Journal of Water and Land Development. No 15 p. 3-18.

Karavan J., Solovej T., YuschenKo Y. 2013. Determination of anthropogenic impact on the Siret River and its tributaries by the analysis of attached algae. Journal of Water and Land Development. No 19 p. 53-58.

ŁASKA G. 2009. Zbiorowiska roślinne siedlisk mokradłowych w dolinie Płoski - ocena aktualnego stanu w zależności od różnych form użytkowania [Plant communities of wetland habitats in the Płoska River valley - an assessment of present status in relation to different forms of land use]. Woda-Środowisko-Obszary Wiejskie. T. 9. Z. 4 (28) p. 141-162.

ŁASKA G. 2012. Różnorodność i walory przyrodnicze zbiorowisk mokradłowych w dolinie Białej, w centrum
Białegostoku [Diversity and natural values of the wetland communities in the Biała valley, in Białystok centre]. Inżynieria Ekologiczna. Nr 29 p. 87-98.

ŁASKA G., KASPRZYKOWSKI Z., KLIK W. 2010. Raport z przeprowadzenia oceny oddziaływania na środowisko dla planowanego przedsięwzięcia polegającego na odbudowie stopnia wodnego „Sarkajmy” wraz z budową elektrowni wodnej w km 12+330 rzeki Sajna, w miejscowości Sarkajmy [Report on environmental impact of the planned project aimed at restoring the dam "Sarkajmy" and construction of hydroelectric power plant in km 12330 of the Sajna River, in a village Sarkajmy]. Białystok. Manuscript pp. 124.

MatuszKIEWICZ W. 2001. Przewodnik do oznaczania zbiorowisk roślinnych Polski [Guide to Polish plant communities identification]. Warszawa. PWN. ISBN 83-0113520-4 pp. 537.

MiodUSZEWSKI W. 2006. Management of water resources in rural areas: the Polish approach. Journal of Water and Land Development. No 10 p. 3-14.

MiODUSZEWSKI W. 2012. Small water reservoirs - their function and construction. Journal of Water and Land Development. No 17 p. 45-52.

MiODUSZEWSKI W., OKRUSZKO T. 2012. Protection of natural wetlands - the examples of conflicts. Journal of Water and Land Development. No 16 p. 35-42.

Natura 2000 undated. Ostoja Warmińska [online]. [Access 20.11.2013]. Available at: http://natura2000.gdos.gov.pl/ data-

files/index/page:11/all:0/province_id14/Ostoja_Warmins ka_sdf.pdf

NowICKI M. 2004. Perspektywy wykorzystania odnawialnych źródeł energii w Polsce [Perspectives of the use of renewable energy sources in Poland]. Aura. Nr 2 p. 12 16.

Rozporządzenie Ministra Środowiska z dnia 9 lipca 2004 r. w sprawie gatunków dziko występujących roślin objętych ochrona gatunkową [Regulation of the Minister of Environment of 9 July 2004 on wild plants species under protection]. Dz.U. 2004. Nr 168 poz. 1764.

Rozporządzenie nr 157 Wojewody Warmińsko-Mazurskiego z dnia 19 grudnia 2008 r. w sprawie Obszaru Chronionego Krajobrazu Doliny Rzeki Guber [Regulation No 157 of Warmia and Mazury Voivode of 19 December 2008 regarding the area of protected landscape of the Guber River valley]. Dz.Urz. Woj.Warm.-Maz. 2008. Nr 198 poz. 3108.

Warda M., Stamirowska-KrZaczeK E., Kulik M. 2013. Floristic diversity of selected plant communities on extensive and abandoned grasslands in the Nadwieprzański Landscape Park. Journal of Water and Land Development. No 19 p.77-82. 


\section{Grażyna LASKA}

Ochrona różnorodności florystycznej doliny rzeki Sajna w aspekcie planowanej budowy elektrowni wodnej w miejscowości Sarkajmy

\section{STRESZCZENIE}

Słowa kluczowe: chronione gatunki roślin, dolina rzeki Sajna, elektrownia wodna, siedliska przyrodnicze Natura 2000

Celem niniejszej pracy jest określenie różnorodności florystycznej doliny rzeki Sajna w bezpośredniej i pośredniej strefie oddziaływania planowanej budowy małej elektrowni wodnej w miejscowości Sarkajmy. Zakres pracy obejmuje analizę zmienności fitosocjologicznej zbiorowisk roślinnych i zmienności florystycznej w świetle oceny potencjalnego wpływu inwestycji na rzece Sajna. Badania prowadzono w 2010 r., wykorzystując metody terenowe połączone z techniką lokalizacji punktów badawczych za pomocą GIS. Łącznie wykonano 73 zdjęcia fitosocjologiczne i dokonano identyfikacji poszczególnych płatów roślinnych, a wykorzystując system globalnego pozycjonowania GPS, dokonano delimitacji zbiorowisk roślinnych i odnotowano stanowiska chronionych gatunków roślin.

W badaniach szaty roślinnej badanego fragmentu doliny Sajny zidentyfikowano 13 zbiorowisk roślinnych, w tym pięć zbiorowisk o znaczeniu wspólnotowym, wymagających ochrony w formie wyznaczenia obszarów Natura 2000. Do grupy tej należą cztery zbiorowiska leśne (Salicetum albo-fragilis, Ficario-Ulmetum, Tilio-Carpinetum, Acer platanoides-Tilia cordata) i jedno zbiorowisko ziołoroślowe (Urtico-Calystegietum). Wśród stanowisk chronionych gatunków roślin odnotowano występowanie jednego gatunku objętego ochroną ścisłą (Daphne mezereum L.) i pięć gatunków roślin objętych ochroną częściową (Viburnum opulus L., Ribes nigrum L., Asarum europaeum L., Eurhynchium angustirete (Broth.) T.J.Kop., Eurhynchium striatum (Schreb. ex Hedw.) Schimp.). Obecność cennych siedlisk przyrodniczych i stanowisk chronionych gatunków roślin w dolinie rzeki Sajna świadczy o szczególnej wartości przyrodniczej i różnorodności biologicznej tego terenu. Zagrożeniem dla ich egzystencji jest planowana budowa elektrowni wodnej, która w wyniku piętrzenia wód przyczyni się do zalewu terasy rzecznej i powstania zbiornika wodnego o powierzchni 7,25 ha. 